Int. J. Dev. Biol. 57: 629-637 (2013)

doi: $10.1387 / \mathrm{ijdb} .130194 \mathrm{hp}$

\title{
Gene targeting in plants: 25 years later
}

\author{
HOLGER PUCHTA* and FRIEDRICH FAUSER \\ Botanical Institute II, Karlsruhe Institute of Technology, Karlsruhe, Germany
}

\begin{abstract}
Only five years after the initiation of transgenic research in plants, gene targeting (GT) was achieved for the first time in tobacco. Unfortunately, the frequency of targeted integration via homologous recombination (HR) was so low in comparison to random integration that GT could not be established as a feasible technique in higher plants. It took another $\mathbf{2 5}$ years and great effort to develop the knowledge and tools necessary to overcome this challenge, at least for some plant species. In some cases, the overexpression of proteins involved in HR or the use of negative selectable markers improved GT to a certain extent. An effective solution to this problem was developed in 1996, when a sequence-specific endonuclease was used to induce a double-strand break (DSB) at the target locus. Thus, GT frequencies were enhanced dramatically. Thereafter, the main limitation was the absence of tools needed to induce DSBs at specific sites in the genome. Such tools became available with the development of zinc finger nucleases (ZFNs), and a breakthrough was achieved in $\mathbf{2 0 0 5}$ when ZFNs were used to target a marker gene in tobacco. Subsequently, endogenous loci were targeted in maize, tobacco and Arabidopsis. Recently, our toolbox for genetic engineering has expanded with the addition of more types of site-specific endonucleases, meganucleases, transcription activator-like effector nucleases (TALENs) and the CRISPR/Cas system. We assume that targeted genome modifications will become routine in the near future in crop plants using these nucleases along with the newly developed in planta GT technique.
\end{abstract}

KEY WORDS: plant biotechnology, gene technology, synthetic nucleases, transformation, double-strand break repair

\section{Introduction}

In this review, we intend to give a concise overview of the history and current developments of GT in plants. Due to space limitations, we will not be able to discuss all of the approaches that have been attempted, especially ones that did not help to improve GT frequencies in the long term. Moreover, we will only discuss the molecular mechanisms of DNA recombination insofar as they are important for the understanding of the GT reaction itself and will primarily refer to recent reviews for further details (LiebermanLazarovich and Levy, 2011; Waterworth et al., 2011). Genome editing in plants was discussed recently in an excellent review (Voytas, 2013), and two chapters on specific aspects of genome editing in plants are also included in this issue (see Marton et al., 2013; D'Halluin and Reuter, 2013). Therefore, we will not go into too much detail on the architecture and set-up of the different types of custom, artificial nucleases that are used for GT as well as their application for targeted mutagenesis.

The production of transgenic plants relies heavily on DNA recombination, which is required in somatic cells to repair DSBs within nuclear DNA. The prevailing mechanism of DSB repair in higher plants is nonhomologous end joining (NHEJ), which is also required for the random integration of foreign DNA into the plant genome. On the other hand, DSB repair can occur by the use of homologous sequences, which depending on the circumstances, occurs with a much lower efficiency than NHEJ (Puchta, 2005).

\section{Mechanisms of homologous double-strand break repair in somatic plant cells}

In principle, it is possible to discriminate between the two different mechanisms of homologous DSB repair, single-strand annealing (SSA) and synthesis-dependent strand annealing (SDSA), in somatic plant cells (Fig. 1). For SSA, a break between two direct repeats is repaired by removing the internal sequences and is thus a non-conservative pathway. SSA appears to be quite

Abbreviations used in this paper: DSB, double-strand break; Cas, CRISPR-associated; CRISPR, clustered regularly interspaced short palindromic repeats; GT, gene targeting; HR, homologous recombination; NHEJ, non-homologous end joining; SDSA, synthesis-dependent strand annealing; SSA, single-strand annealing; TALEN, transcription-activator like effector nuclease; ZFN, zinc finger nuclease.

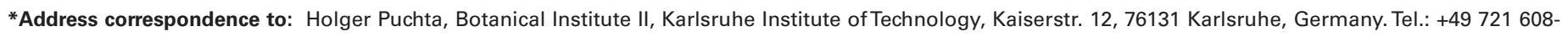
43833; Fax.: +49 721 608-44874; e-mail: holger.puchta@ kit.edu 
efficient in genomic regions with tandemly arranged duplication; in these regions, up to one out of every three DSBs is repaired by this mechanism (Siebert and Puchta, 2002). In contrast, SDSA is conservative. A homologous sequence is copied into the break site without altering the donor. SDSA appears to be approximately five to ten times less efficient than SSA under comparable conditions (Orel et al., 2003). In both pathways, single-stranded overhangs are produced via exonuclease-catalyzed resection after DSB induction. In the case of SSA, overhangs at both ends of the break carry complementary sequences, and the two single-strands can directly anneal with one another to form a chimeric DNA molecule. If the molecule contains 3' overhangs, these will be trimmed; otherwise, single-stranded regions would be filled in by repair synthesis. In the case of SDSA, a 3' end invades a homologous double-strand forming a D-loop. Repair synthesis begins using the newly paired strand as a template. After elongation, the strand is displaced from the D-loop structure and anneals with the 3' homologous strand that becomes available due to resection of the second end of the DSB. Thus, gene conversion without a loss of sequence information is the final result of the reaction (Fig. 1). Not surprisingly, the involvement of DNA repair proteins differs considerably between

SSA

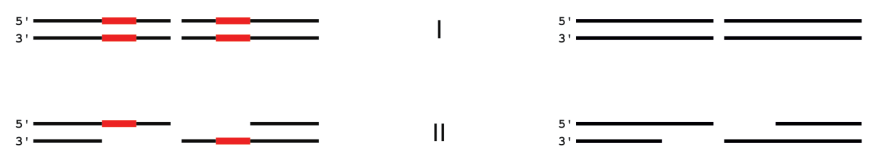

III

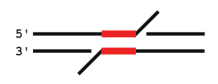

IV

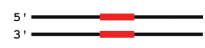

SDSA

VI
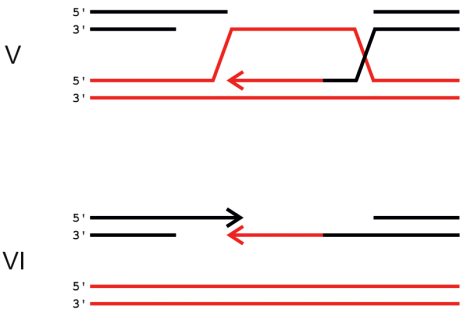

VII$$
\text { (:) }
$$

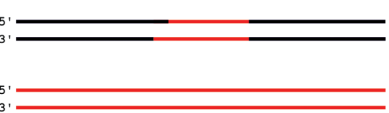

Fig. 1. Mechanisms of homologous double-strand break repair in somatic plant cells. Models of the SSA and the SDSA pathway of recombination are depicted. The HR reaction is initiated by the induction of a DSB (I), and resection occurs to produce free $3^{\prime}$ ends (II). In the case of SSA, homologies within the SSDNA regions can be used for the direct annealing of the two strands (III). After trimming the intermediate, the integrity of the dsDNA molecule is restored, leading to a deletion between the homologous regions (IV). Due to the loss of genetic information, the SSA-mediated repair pathway is regarded as a non-conservative mechanism. In the case of SDSA, a free 3' end invades another dsDNA molecule that acts as donor of genetic information and is homologous to the break site (V). After a copying process is initiated, the $3^{\prime}$ end is elongated using the dsDNA matrix. Later, the $3^{\prime}$ end is set free and reanneals with the second strand at the break site (VI). The resulting dsDNA molecule contains two gaps that are repaired by DNA fill-in synthesis. Thus, a dsDNA molecule is restored without loss of genetic information. Therefore SDSA can be regarded as a conservative mechanism. these two pathways. Proteins involved in the strand exchange reaction, such as AtRAD51 or AtRAD54, are required for SDSA but not for SSA (Roth et al., 2012). In contrast, certain helicases such as AtRECQ4A and nucleases such as AtMUS81 that play a role in SDSA might also play a minor role in SSA (Mannuss et al., 2010), although no factor that is specific for SSA has been characterized yet.

\section{Gene targeting in plants - the first experiments}

Whereas DNA integration in bacteria and yeast occurs primarily via $\mathrm{HR}$ and genes can be readily targeted with homologous sequences, the situation in most multicellular organisms is quite different. GT in higher plants and animals was difficult to accomplish for a long time. Due to the pioneering and Noble Prize-wining work of Smithies (Doetschman et al., 1987) and Capecchi (Thomas and Capecchi, 1987), GT in mouse embryonic stem cells was accomplished successfully more than two decades ago. Nevertheless, similar success was not achieved in other higher eukaryotes for many years.

At the Friedrich Miescher Institute in Basel, Switzerland, Jurek Paszkowski was the first to demonstrate in his pioneering study that genes can indeed be targeted in plant cells (Paszkowski et al., 1988). By direct gene transfer to tobacco protoplasts, he demonstrated that plasmid DNA containing part of the kanamycin gene could be integrated into the genome at a low frequency via HR such that a complementary gene fragment was restored. In addition to direct gene transfer, Paul Hooykaas and his group were able to demonstrate that GT in plant cells by Agrobacterium-mediated TDNA transformation worked as well (Offringa et al., 1990). However, in these and a number of subsequent studies, the observed GT frequencies remained disappointingly low, at approximately one targeting event per $10^{4}$ to $10^{5}$ transformation events using different transformation methods and plant species [for a review of the first 15 years of GT experiments, see (Puchta, 2002)].

Interestingly, different groups analyzed the few targeting events that were isolated with selectable markers as model substrates and revealed that HR did not occur in all cases at both end of the targeting vector during the recombination reaction [e.g. (Hanin et al., 2001)]. In principle, two different classes of events could be identified, in which one end of the vector recombined with the target via HR and the other was repaired via NHEJ (Fig. 2). In one class, the targeting vector was inserted at a site of homology ("one-sided events"). Thus, the vector was inserted at the target locus with one junction formed via HR and the other via NHEJ. However, there was a second class of events in which the selectable maker gene was restored by copying parts of the genomic sequence onto the targeting vector, which was then integrated elsewhere in the genome via NHEJ ("ectopic GT events"). Thus, although the marker gene was restored via HR using the target locus as template, the original target was not changed and was still present in the genome. Although the outcome of these reactions improved our understanding of the mechanisms of recombination, they fell short of the biotechnological expectation of knocking out a gene in a controlled manner.

\section{Manipulation of the enzyme machinery can help (a bit)}

If GT is not efficient in plants, why not transfer into plants com- 


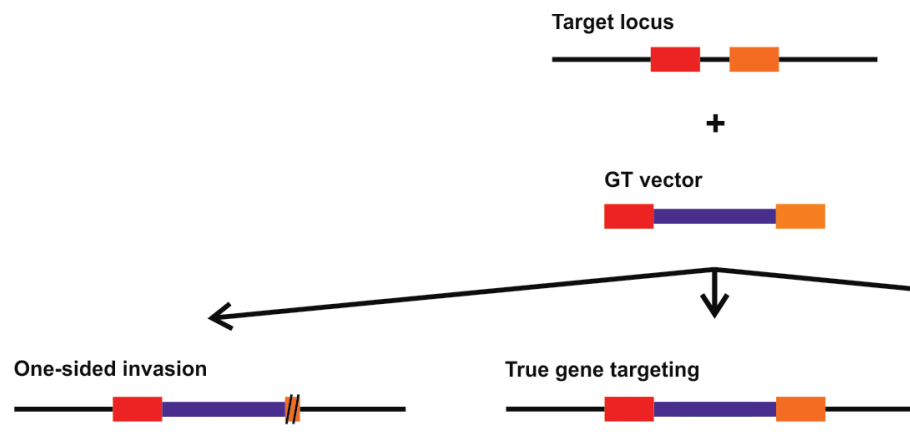

ponents of the HR enzyme machinery from an organism in which GT works well? Several groups have addressed this possibility, and we would like to note the two most prominent examples, the RecA gene of Escherichia coli and the RAD54 gene of Saccharomyces cerevisiae. The group of Bernd Reiss produced transgenic tobacco plants expressing RecA, the strand exchange protein and key HR factor from bacteria. As expected, the rate of intrachromosomal $\mathrm{HR}$ was enhanced by one order of magnitude in plants expressing the E. coli enzyme. Unfortunately, no significant enhancement of the GT frequency could be achieved with Agrobacterium-mediated transformation (Reiss et al., 2000). In a different approach, the group of Avi Levy overexpressed the RAD54 gene of baker's yeast in Arabidopsis. ScRAD54 is required for efficient strand invasion of HR intermediates. Indeed, the ScRAD54 protein substantially enhanced GT frequency. The authors used a promoterless GFP ORF inserted in an Arabidopsis cruciferin gene as assay system. As Cruciferin is a seed storage protein, integration of the GFPcontaining gene into the genomic target site produced fluorescent seeds, making it a convenient and non-destructive marker for GT (Shaked et al., 2005). The same authors expanded on their work in a later study with egg-cell specific expression of RAD54 to enhance GT using the Agrobacterium infiltration technique for transformation (Even-Faitelson et al., 2011).

In addition to using enzymes from other organisms, the manipulation of the intrinsic recombination machinery of plants could also enhance GT efficiency. It is possible that NHEJ and HR function in equilibrium and that GT could be enhanced drastically by blocking NHEJ. However, no convincing study applying this approach to plants has been published thus far. It has been shown recently that these difficulties may be caused by the existence of independent end-joining pathways in plant cells (Charbonnel et al., 2011). A mild reduction in T-DNA transformation efficiencies was reported when single NHEJ factors were knocked out, but no GT experiments have yet been published concerning this issue (Friesner and Britt, 2003; Nishizawa-Yokoi et al., 2012).

Indeed, mutation of certain host factors involved in DNA repair can also lead to a hyper-recombination phenotype, as measured by increased intra- or interchromosomal HR frequencies. This process has been confirmed for certain DNA helicases such as AtREC4QA or AtFANCM that are involved in the control of HR (Hartung et al., 2007; Knoll et al., 2012) and for chromatin assembly factors such as AtCAF1 (Endo et al., 2006; Kirik et al., 2006). Again, no GT experiments have been published using these mutant backgrounds.
Fig. 2. Different outcomes for gene targeting experiments in plants. $H R$ of the target locus with the GT vector leads to the desired gene replacement (middle). In addition to the desired insertion, two other types of recombinants were often found. After one end of the incoming DNA had recombined with the target locus via $H R$, the other end could integrate at the target locus via NHEJ lone-sided invasion, left). Alternatively, the transgene might integrate elsewhere in the genome after $H R$ at one end without changing the target gene (ectopic GT, right).

The major problem with approaches relying on the manipulation of the recombinatorial enzyme machinery is that these strategies may destabilize the genome of an organism in a general way. One must bear in mind that if $\mathrm{HR}$ is more efficient, than enhanced recombination might occur not only between the targeting vector and the target locus but also between repetitive sequences all over the genome, leading to undesired and uncontrolled off-site effects. Attempts have been made to minimize the time window for GT using inducible or organ-specific promoters (Even-Faitelson et al., 2011), but even with these approaches, exclusive activation of the site of interest has not been possible.

\section{Gene targeting in Physcomitrella patens remains a mystery}

In contrast to flowering plants, the moss Physcomitrella patens is able to integrate DNA efficiently via HR (see Strotbek et al., 2013 in the current issue). This finding was first reported by Didier Schaefer and Jean-Pierre Zryd (Schaefer and Zryd, 1997). Soon thereafter, Ralf Reski's group demonstrated that this technique could efficiently knock-out gene functions (Strepp et al., 1998). This moss has become a valuable system for the investigation of the basic processes of plant biology. Nevertheless, no obvious peculiarities in the DNA repair and recombination machinery were found, despite the fact that the genome sequence of this moss was elucidated some years ago (Rensing et al., 2008). Thus, transferring the efficient GT machinery of Physcomitrella to higher plants has not yet been accomplished because we do not currently understand why GT is so efficient in moss.

\section{Negative selection can help (a bit)}

Another strategy to eliminate surplus random integration events is to not block the NHEJ pathways or tune up the HR machinery but to simply select against random NHEJ events, so that these are eliminated from the transgenic pool. Negative selectable marker genes can be used for this approach. These genes have to be included in the targeting construct flanking the homologous regions so that they will not be integrated into the genome if HR occurs correctly (Fig. 3). By targeting the waxy gene of rice, Shigeru lida's group was able to demonstrate in a pioneering study from a decade ago that GT experiments can be performed in plants using a negative selectable marker (Terada et al., 2002). They performed 
Agrobacterium-mediated transformation of highly proliferative callus material derived from the seeds of Oryza sativa with a T-DNA carrying genomic homologies of several kbs. The homologies were flanked by two genes for diphtheria toxin, which are lethal to plants when randomly integrated into the genome. Thus, recombinant calli could be produced and regenerated to fertile plants as well. The estimated ratio of targeted to random integration events was $6.5 \times 10^{-4}$, which makes this technique feasible but laborious. Since then, the group succeeded in targeting a few other natural genes in rice (Ono et al., 2012), indicating that the technique is a useful way to achieve GT in plants. Nevertheless, in the long term, the negative selection strategies might be outcompeted by less time-consuming approaches linked with DSB-induced GT in plants (see below).

\section{Double-strand break induction: a major breakthrough for site-specific integration}

In the end, what helped to solve the GT challenge in plants was to simply copy nature. An efficient way to induce recombination at specific genomic loci is to break both strands of the DNA at the site of interest in a genetically programmed way, as occurs during meiosis or during mating type switching. Under such circumstances, DSBs are often induced by specific cellular enzymes. Indeed, it was demonstrated some time ago in yeast that artificial sites can also be activated for HR by the induction of DSBs [for a review, see (Paques and Haber, 1999)].

Therefore, site-specific nucleases became an obvious possible strategy to increase the HR frequency in plants. Obviously, for this purpose, endonucleases are required with restriction sites that are complex enough that, statistically, no natural site should be present in the respective host genome to avoid unwanted offtarget effects. A promising candidate is the homing endonuclease ("meganuclease") I-Scel, which was originally isolated from yeast mitochondria. It has a 18-mer recognition site (Fig. 4A). The I-Scel ORF integrates itself by inducing a DSB into the mitochondrial $21 \mathrm{~S}$ rRNA gene. The DSB is repaired with the aid of a copy of the 215 rRNA gene that already includes the I-Scel ORF as an intron. Thus I-Scel is able to spread in the mitochondrial DNA pool (Jacquier and Dujon, 1985). Initially, the applicability of DSB-induced HR to plants was confirmed using I-Scel and transiently transformed plasmid molecules in Nicotiana protoplasts (Puchta et al., 1993). Most importantly, a DSB could also be introduced at a transgenic locus within the tobacco genome in vivo via transient expression of I-Scel, resulting in a homologous integration frequency of up to $10^{-2}$ at the transgenic locus using a T-DNA carrying homologies to this locus (Puchta et al., 1996). In most of the events, both ends of the target vector were integrated via HR, but combinations of HR and NHEJ were also found. Using the same system, it was also demonstrated that GT frequencies were approximately half as high if a T-DNA only carried homology to one end of the break (Puchta, 1998). This is a strong indication that HR is initiated at both ends independently, which is in accordance with the SDSA model. However, even without homologies to the genomic target site, it was found that T-DNAs could insert into meganuclease-induced DSBs via NHEJ (Chilton and Que, 2003; Salomon and Puchta, 1998; Tzfira et al., 2003). Moreover, meganuclease-mediated DSB induction could also be used for the excision of sequences that were flanked by the respective meganuclease recognition sites

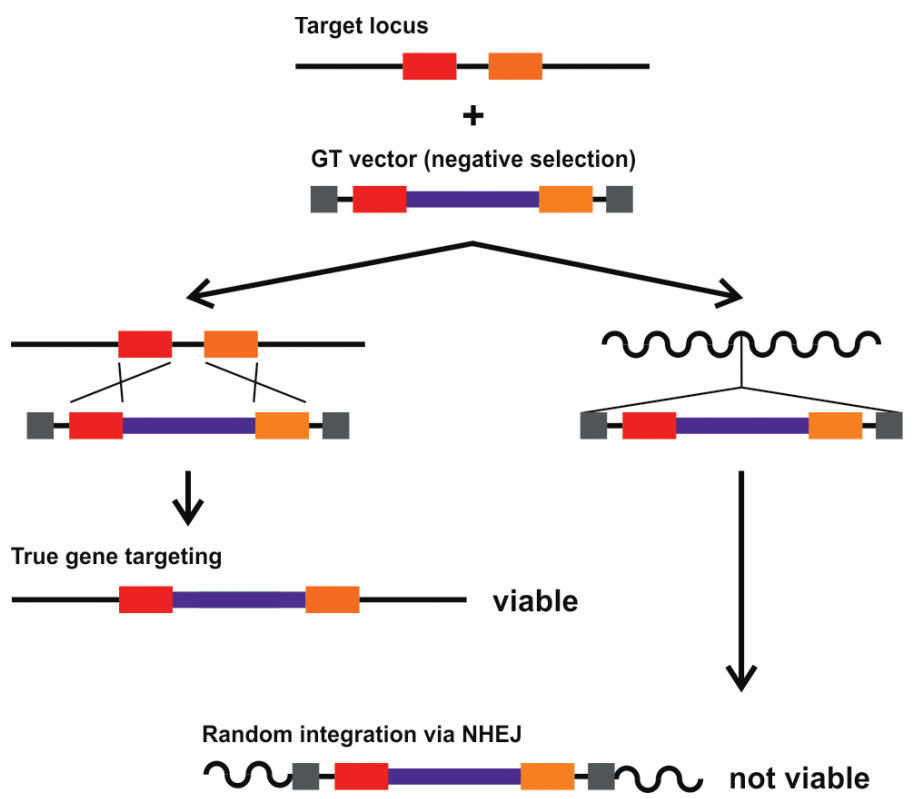

Fig. 3. The principle of negative selection to enhance gene targeting frequencies. At both ends of the DNA used for transformation (GT vector), a negative selectable marker (depicted as black box) is cloned outside of the homologous regions used for targeting. In the case of "perfect" GT events, both copies of the marker are eliminated (left). In the cases of random integration, all types of one-sided targeting or ectopic recombination, both or at least one negatively selectable marker cointegrates with the transgene. When negative selection is applied, only cells without such a marker are able to survive, which leads to a massive enrichment of true GT events in the cell population.

from the genome either via NHEJ or, in case of available homologies, by SSA (Siebert and Puchta, 2002). Additionally, the loss of gene function via NHEJ after DSB induction was also achieved (Kirik et al., 2000).

Although early studies with homing endonucleases demonstrated how efficiently sequence-specific meganucleases could be used for plant genome editing, the limitation of having only one particular sequence as a recognition site precluded any practical application for the knock-out of natural genes. It was not possible to target DSBs to sites of interest in the genome. For this purpose, nucleases that were prone to artificial manipulation of their binding sites were required. Therefore, more than a decade ago, attempts to develop meganucleases with artificially modulated binding sites were initiated (Fig. 4B). Although such an enzyme was recently used for the NHEJ-mediated targeted mutagenesis of maize (Gao et al., 2010), no successful GT by a modified meganuclease has been published for plants. In general, the problem with manipulating meganuclease binding sites is that the recognition specificity has to be changed without harming the endonuclease activity, although both reside in the same domain. Therefore, alternative approaches were taken relatively early to combine an endonuclease domain with a separate DNA binding domain of different biological origin that could be manipulated independently. An endonuclease domain from a partial ORF of the classic restriction enzyme Fokl, which acts as dimer, has been used. With this system, the dimerization of two Fokl domains through the action of two independent DNA binding domains is necessary to induce a DSB. As both DNA bindings domains can be manipulated independently to recognize 
different sequence tracks in close proximity on opposite strands, the specificity of the artificial enzyme can be dramatically increased. Two types of DNA binding domains have been used successfully (Fig. 4 C,D). More than a decade ago, work on zinc finger nuclease (ZFNs) was initiated, and in the last few years, Transcription Activator-like Effector Nucleases (TALENs) have garnered interest. In the following sections, the progress with these systems and their application to GT in plants will be discussed in detail.

\section{Zinc finger nucleases: fulfillment of the promise}

The set-up of ZFNs was pioneered by Dana Carroll. ZFNs consist of the endonuclease domain of the restriction enzyme Fokl fused to the zinc finger binding arrays found in transcription factors (Smith et al., 2000). A zinc finger binding array typically recognizes three consecutive nucleotides. Three to four arrays are combined as DNA binding sites so that, in total, 18 to 24 bases of genomic sequence can be recognized per dimer (Fig. 4C). Dan Voytas recognized early on the potential of these enzymes for genome
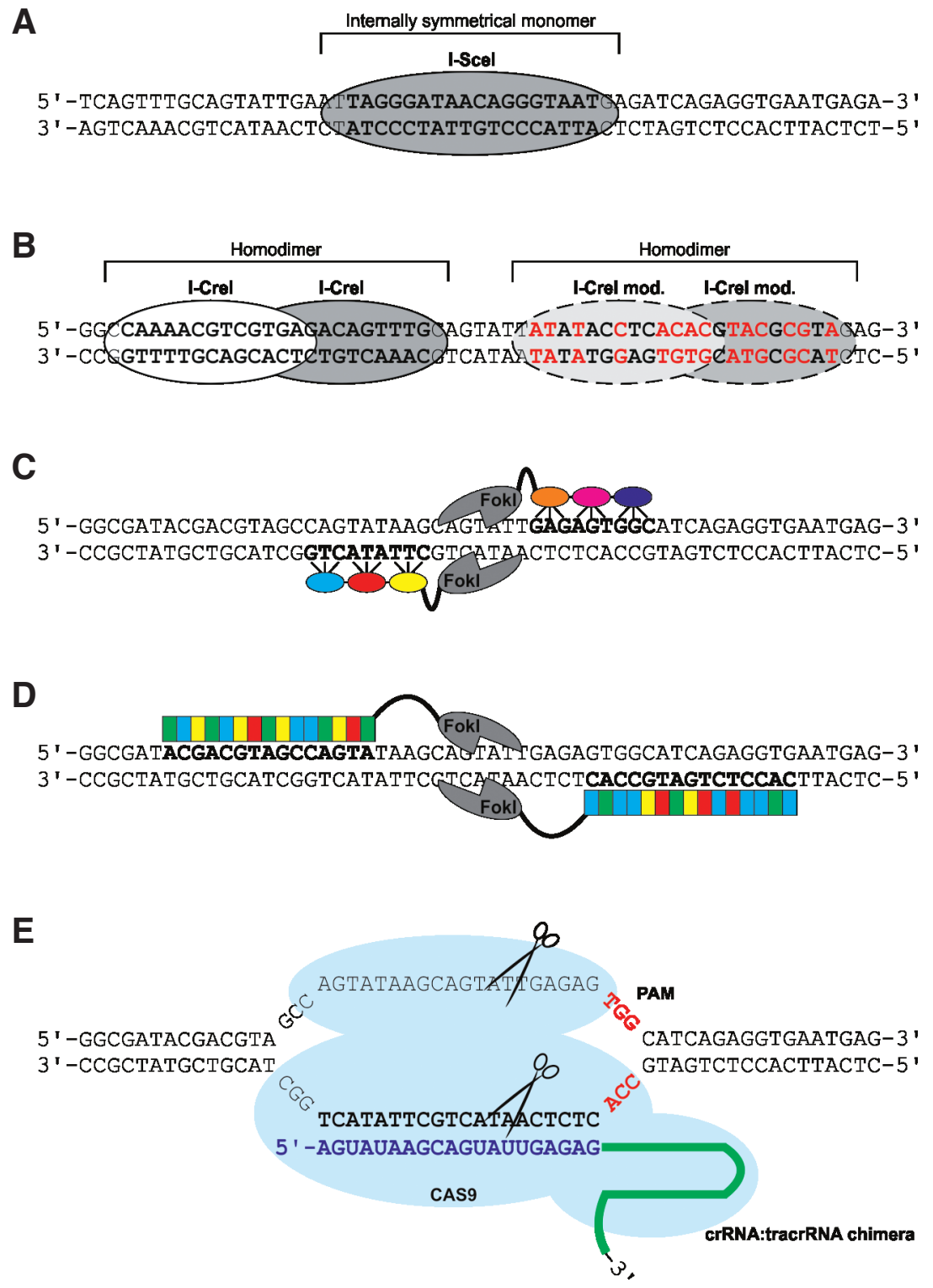

Fig. 4. Different types of nucleases used for the induction of genomic double strand breaks. (A): The monomeric homing endonuclease I-Scel: this enzyme is only able to recognize and cut a specific 18-bp recognition site. (B) The dimeric homing endonuclease I-Crel: on the left is the wild type enzyme with its specific 22-bp recognition site, and on the right is a derivative with an artificially modified binding site. (C) ZFN dimer: each subunit consists of a FokI nuclease domain and a zinc finger domain that can be manipulated and is built out of arrays that bind three consecutive bases each. By changing individual arrays, the binding specificity can be changed, whereas the length of the binding site can be varied by changing the number of arrays. (D) Dimeric TALEN: each subunit consists of a FokI nuclease domain and a TALE DNA binding domain that can be manipulated and is built out of repeats that are specific for individual bases. Binding specificity is manipulated by combining repeats that recognize individual bases in different orders. (E) The CRISPR/Cas System: whereas the Cas9 protein is responsible for guiding the sgRNA (crRNA:tracrRNA) and the cleavage of both strands of the target DNA, binding specificity is defined solely by the chimeric crRNA:tracrRNA molecule. Within this RNA, a stretch of 20 bases is complementary to the respective target site. The specificity can be changed by modifying this sequence motif as long as the correct (NGG) protospacer-adjacent motif (PAM) is present. By taking the PAM into account, any genomic sequence can in principle be targeted. 
(Voytas, 2013). Off-target effects are a source of some concern. ZFNs might also cut other sites in the genome that are similar to the target site, inducing unpredicted mutations that might result in uncontrolled secondary effects. Indeed, a collection of ZFNs had negative effects on cell proliferation, which may indicate that these nucleases could harm cells by creating unwanted DSBs at secondary sites. Initially, the construction of ZFNs was quite time consuming. Although kits for the construction of ZFNs are now available, construction is still quite laborious. A further uncertainty stems from the fact that the different binding modules in the zinc finger binding arrays influence each other. Therefore, the construction of domains for new genomic sites has not always been as predictable and efficient as expected.

\section{Transcription activator-like effector nucleases make things easier}

In a groundbreaking analysis, the plant pathologists Ulla Bonas and Jens Boch discovered that a protein delivered into its host by the bacterial pathogen Xanthomonas carries a DNA binding domain that binds to different plant promoters (Boch et al., 2009). The domain consists of 13 to 28 copies of highly conserved repeats spanning 34 amino acids each. Interestingly, each repeat is able to recognize a single base. Thus, the scientific community acquired an alternative and easily programmable DNA binding domain. Very quickly, several proofs of concept were reported by various groups, who documented that a powerful new class of nucleases was feasible with a set-up similar to ZFNs (Voytas, 2013) (Fig. 4D).

Due to the repetitive nature of the DNA binding motifs, cloning of TALENs in E. coli could potentially cause problems. However, the development of ligation-based methods made it possible to handle the assembly more easily. TALENs can now be assembled within a week in the lab using publically available kits (Cermak et al., 2011)". Moreover, as every single base is addressed by a single repeat, the management of binding specificities is also much easier relative to ZFNs. Thus, TALENs to almost every possible sequence motif in the genome can be produced without experimental difficulty and with a high success rate. Due to the longer recognition sites of TALENs, these nucleases may cause fewer unwanted off-target effects than ZFNs.

As TALENS are a relatively recent development and plants have a relatively long generation time, few reports have been published thus far documenting the successful application of these enzymes for genome editing in plants. In one study, the authors demonstrated that the binding motif for a pathogen-based transcription factor could be destroyed by TALEN-mediated introduction of a mutation into the promoter of the OsSWEET14 gene, leading to enhanced disease resistance in rice (Li et al., 2012). The group of Dan Voytas introduced targeted mutations within the ALS gene in up to $30 \%$ of transformed tobacco protoplasts using TALENs. The same authors designed a TALEN-mediated GT experiment using a donor template that created an in-frame gene fusion between ALS and a YFP marker gene. GT efficiency was measured by quantifying YFP fluorescence using flow cytometry. Approximately $14 \%$ of protoplasts showed fluorescence, indicating a very high frequency of GT. In the same study, GT experiments were performed with a TALEN and a 322-bp donor molecule differing by 6-bp from the ALS coding sequence. Even without selection, $4 \%$ of the regenerated calli showed evidence of targeted gene replacement (Zhang et al.,
2013). These results strongly indicate that TALENs are indeed the most convenient and most effective tools to perform DSB-induced GT in plants today.

\section{RNA guided double-strand break induction: another breakthrough?}

Just this year, a bacterial system based on sequence-specific recognition via an RNA molecule for the manipulation of eukaryotic genomes was described: the CRISPR/Cas System (clustered regularly interspaced short palindromic repeats (CRISPR) / CRISPRassociated) [for a review, see (Mussolino and Cathomen, 2013)]. This system relies on the bacterial endonuclease Cas9, which can be redirected to different target sites simply by modifying the sequence of a single synthetic chimeric guide RNA (sgRNA or crRNA:tracr RNA) (see Fig. 4E). This new technique has been applied to genome editing in a variety of different eukaryotes including plants (Feng et al., 2013; Li et al., 2013; Nekrasov et al., 2013; Shan et al., 2013; Xie and Yang, 2013) and appears to be as efficient as TALENs. What makes the system especially attractive is the very simple design process. One needs only insert the desired DNA oligonucleotide into a vector construct for target site selection, as specificity is solely defined by base-complementarity to the guide RNA. The Cas9 protein does not require any reengineering and has worked well for all of the target sites that have been studied. Moreover, expressing multiple guide RNAs allows multiplexing, which reduces the costs and time needed to generate plants with multiple targeted mutations.

\section{In planta gene targeting}

The availability of various tools for site-specific DSB induction makes GT a feasible task, at least for plant species that are easy to transform and regenerate. Unfortunately, this is still not true for most cultivated plant species. For many crop plants, it is still difficult to obtain and regenerate to fertility even a single transgenic line. Therefore we recently developed a GT technique that should be applicable to all transformable plant species, even if the transformation efficiency is extremely low (Fauser et al., 2012). The basic idea behind the strategy is that, if one were able to perform the targeting reaction during plant development, then the progeny should carry targeted modifications. Initially, an efficient variation of in vivo GT was developed in Drosophila melanogaster by the group of Kent Golic more than a decade ago (Rong and Golic, 2000). Their method relied on the integration of the targeting vector into the host genome. By induced expression of a site-specific recombinase, a circular DNA was excised from the genome. This circle was then linearized by the simultaneous expression of a homing endonuclease. Thus, GT could be achieved by means of a linearized DNA vector. The set-up of this system was quite complex, because in addition to the construction of the donor sequence with sites for the recombinase and the homing endonuclease, expression cassettes for both enzymes have to be included into the transgene construct or supplied in trans. No successful use of such a system has been reported in plants thus far. Nevertheless, an in vivo approach did not seem unattractive for plants, if it would be possible to simplify the procedure. Therefore, we developed an alternative method using Arabidopsis as a model. Although the scorable marker $\beta$-glucuronidase (GUS) and I-Scel were used, the 


\section{Integration}
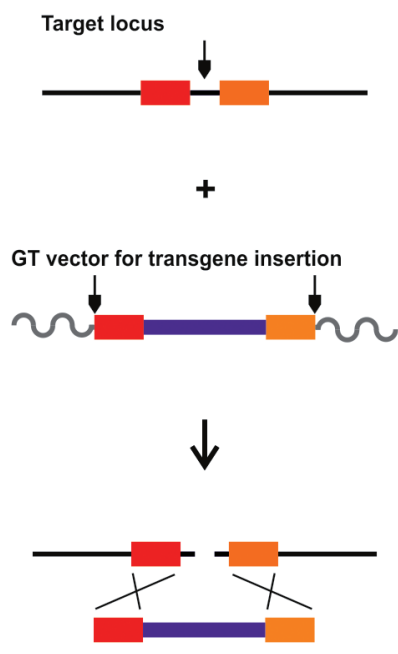

$\downarrow$

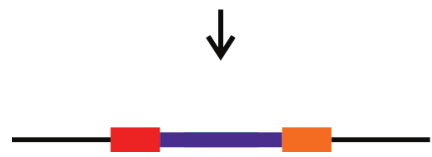

Modification

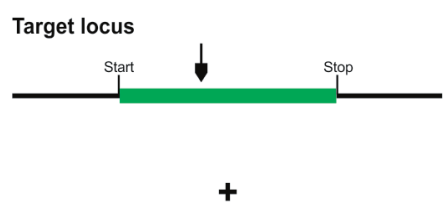

GT vector for AS exchange
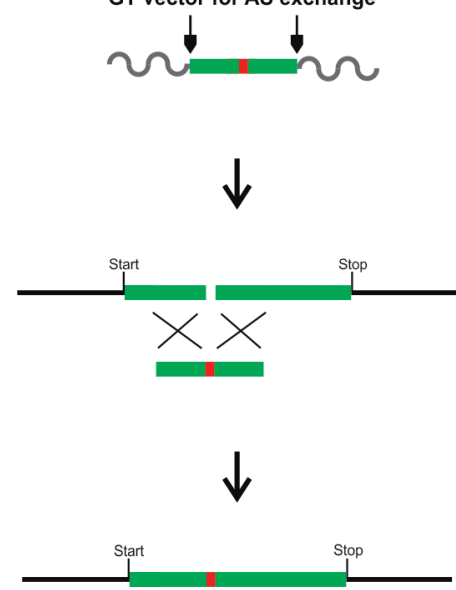

Fig. 5. In planta gene targeting. In addition to the construction of a custom-made nuclease that is able to induce a DSB at a unique genomic site of interest, a transgene construct is produced that harbors the targeting vector as a cassette flanked by two restriction sites of the same nuclease. The targeting vector might contain a gene between the homologous regions that can be used for crop improvement (left) or just a defined modification of one or a few amino acids. After random integration in the plant genome, expression of the endonuclease is induced. This could be achieved by placing the nuclease ORF under the control of an inducible or organ-specific promoter in the same targeting vector outside regions of homology or by expression in trans either by transient or stable transformation. The GT vector is excised and the target locus is activated simultaneously by DSB induction. The vector integrates via HR into the target, leading to a stably modified endogenous locus. Each arrow depicts a recognition site for the nuclease.

method should be applicable to any endogenous locus and custommade nuclease. The basic principle of the in planta GT technique is depicted in Fig. 5. The system is based on a transgene carrying sequences homologous to the target locus that are flanked by two recognition sites for a custom-made endonuclease that also cuts the locus of interest. Expression of the enzyme, with the help of an inducible, organ-specific or constitutive promoter, leads to the simultaneous release of a linear GT vector and the induction of a DSB at the target locus. Thus, GT can take place in all cells during vegetative growth whenever the enzyme is expressed either based on stable or transient transformation. Events that occur in the shoot meristem or late in development could be transferred to the germline. As a consequence, GT events can be harvested as seeds. In the model system, GT during somatic growth was scored by GUS expression, whereas GT events entering the germline could be detected after harvest and the germination of seeds. Here, seedling that uniformly expressed GUS could be detected. For different target/donor combinations, up to one GT event per 100 seeds could be recovered. Thus, hundreds of seeds with a GUS gene restored by GT could be obtained.

Molecular analysis of recombinant lines indicated that HR occurred at both ends of the DSB in almost all of them (19 out of 20 tested). Additionally, no extra copies of the vector were integrated elsewhere in the genome. Whereas classical GT approaches rely on the generation of larger numbers of transformation events, in planta GT requires, in principle, a single transformation event with the GT vector. Upon expression of the endonuclease, the GT vector is likely set free during the life cycle of a plant in a large percentage of cells. As is the case for a hemizygous, single-copy transgene, only one copy of the target vector can be set free per genome, so the number of unwanted random integration events is minimized, in contrast to classical GT approaches in which multiple copies of a vector are often transferred into a single cell. As the donor locus can be segregated from the targeted integration site, the plant that ends up in the field carries only the designed change in the target without any additional transgene sequences. It will be interesting to see how easily this concept can be applied to crop plants in the future. A first indication that this may be possible comes from a recent study in maize with a similar system that used marker genes and the endonuclease I-Scel. The authors demonstrated that also an ectopic copy of the targeting vector could be used as a template after DSB induction at the target locus (Ayar et al., 2012).

\section{Outlook}

The last few years saw a tremendous acceleration in the development of new tools for genome editing. Many options that would have been difficult to envision a few years ago now seem to be possible. Naturally, the application of genome editing tools is more advanced in the animal field as in plants due to its tremendous potential in medical applications. Nevertheless, there have been a series of consequences that are of special importance for plants and agriculture. On the one hand, we should not be too optimistic: GT is far from being established in many plant species, and the plant community will still need to spend a tremendous amount of time and expend effort to change this situation. It might well be that, under certain circumstances, only some combinations of custom-made endonucleases, the manipulation of the enzyme machinery and the use of negative selection will lead to feasible GT frequencies in some crop species.

Moreover, due to the recent developments, we not only have many more tools in our hands for genome editing but also must reevaluate our strategies to define which tool is optimal for a specific purpose. For years, GT seemed to be the only way to knock-out gene functions in a directed way. However, as was demonstrated with ZFNs and TALENs, DSB induction and repair via NHEJ in many cases leads to the complete knock-out of gene function. It is technically less demanding to just induce a DSB without simultaneously offering a repair template to the cell. The DSB inducing reagent can be supplied as DNA but also as RNA or even as protein. This type of method has been used successfully for mutant production not only in Arabidopsis but also in several important crop species. Therefore, we expect that future applications of DSB-induced GT 
will be restricted to two main tasks: the site-specific integration of foreign genes and the introduction of sophisticated changes, such as the modification of single or a few amino acids in plant genes to modify their function in a controlled way (Fig. 5).

There are multiple ways in which site-specific integration of genetic information will improve our ability to address specific questions in basic science and biotechnology. New sequence information can be targeted to specific regions of the genome. Thus, it will be possible in a very systematic way to discriminate between sequence content and genomic position. Discriminating between these levels is important for different research areas such as gene expression, DNA repair, meiotic recombination and epigenetic modifications. However, the approach is also extremely valuable for agriculture: stacking improved traits at specific loci ("landing pads") in elite cultivars of crop plants is of central interest to plant breeding companies.

The same holds true for using DSB-induced GT to change single or small stretches of amino acids in proteins or single or a few base pairs in DNA control elements. Fine-tuning of genetic information in the original genomic context will allow us to identify connections between enzyme structure and function and modify enzyme expression or specificity. Techniques that create small changes of a few base pairs in the genome also raise the question of how such an organism should regarded in relation to our current understanding of "genetically modified organisms" (GMOs). GMOs are generally regarded as organisms that carry DNA from another species ("foreign" DNA). On the contrary, plants modified by chemical mutagens, gamma radiation or X-rays in breeding programs are regarded as "natural" varieties, although they harbor a heavy mutational load. Taking plant genome sizes into account, statistical basics indicate that a small stretch of deleted or modified DNA sequence at its endogenous locus cannot be regarded as "foreign". Therefore, there is no rational argument for considering plants that have small changes caused by DSB-induced GT or NHEJ to be GMOs. This example demonstrates how far-reaching the consequences of these new genome editing techniques are. Public concerns about the use of GMOs in agriculture might become obsolete in the long run.

\section{Acknowledgements}

HP wants to thank all of the colleagues with whom he has directly or indirectly shared efforts to develop genome editing in plants over the last two decades. We regret that the majority of publications dealing with DSB repair and GT in plant systems could not be cited due to space limitations. Moreover, we would like to thank Manfred Focke for his critical reading of the manuscript. Work on GT in our group has been funded over the years by the European Union and the Bundesministerium für Bildung und Forschung (BMBF).

\section{References}

AYARA, WEHRKAMP-RICHTERS, LAFFAIRE J B, LE GOFFS, LEVY J, CHAIGNON S, SALMI H, LEPICARD A, SALLAUD C, GALLEGO M E et al., (2012). Gene targeting in maize by somatic ectopic recombination. Plant Biotechnol $J 11: 305-314$.

BOCH J, SCHOLZE H, SCHORNACK S, LANDGRAF A, HAHN S, KAY S, LAHAYE T, NICKSTADT A, BONAS U (2009). Breaking the code of DNA binding specificity of TAL-type III effectors. Science 326: 1509-1512.

CERMAK T, DOYLE EL, CHRISTIAN M, WANG L, ZHANG Y, SCHMIDT C, BALLER JA, SOMIA NV, BOGDANOVE AJ and VOYTAS DF (2011). Efficient design and assembly of custom TALEN and other TAL effector-based constructs for DNA targeting. Nucl. Acids Res. 39: e82.
CHARBONNEL C, ALLAIN E, GALLEGO M E, WHITE C I (2011). Kinetic analysis of DNA double-strand break repair pathways in Arabidopsis. DNA Repair (Amst) 10: 611-619.

CHILTON M D, QUE Q (2003). Targeted integration of T-DNAinto the tobacco genome at double-stranded breaks: new insights on the mechanism of T-DNA integration. Plant Physiol 133: 956-965.

DE PATER S, PINAS J E, HOOYKAAS PJ,VAN DER ZAALBJ (2012). ZFN-mediated gene targeting of the Arabidopsis protoporphyrinogen oxidase gene through Agrobacterium-mediated floral dip transformation. Plant Biotechnol J11:510-515.

D'HALLUIN, K. and RUITER, R. (2013). Directed genome engineering for genome optimization. Int. J. Dev. Biol. 57: 621-627. [DOI: 10.1387/ijdb.130217kd].

DOETSCHMANT, GREGG RG, MAEDAN, HOOPERML, MELTONDW, THOMPSON S, SMITHIES O (1987). Targetted correction of a mutant HPRT gene in mouse embryonic stem cells. Nature 330: 576-578.

ENDO M, ISHIKAWAY, OSAKABE K, NAKAYAMA S, KAYAH, ARAKIT, SHIBAHARA K, ABE K, ICHIKAWA H, VALENTINE L et al., (2006). Increased frequency of homologous recombination and T-DNA integration in Arabidopsis CAF-1 mutants. EMBO J 25: 5579-5590.

EVEN-FAITELSON L, SAMACH A, MELAMED-BESSUDO C, AVIVI-RAGOLSKY N LEVY A A (2011). Localized egg-cell expression of effector proteins for targeted modification of the Arabidopsis genome. The Plant journal: for cell and molecular biology 68: 929-937.

FAUSER F, ROTH N, PACHER M, ILG G, SANCHEZ-FERNANDEZ R, BIESGEN C PUCHTA H (2012). In planta gene targeting. Proc. Natl. Acad. Sci. USA 109: 7535-7540.

FENG, Z., ZHANG, B., DING, W., LIU, X., YANG, D.L., WEI, P., CAO, F., ZHU, S., ZHANG, F., MAO, Y. et al. (2013). Efficient genome editing in plants using a CRISPR/Cas system. Cell Res. 23: 1229-1232.

FRIESNER J, BRITT A B (2003). Ku80- and DNA ligase IV-deficient plants are sensitive to ionizing radiation and defective in T-DNA integration. Plant J 34: 427-440.

GAO H, SMITH J, YANG M, JONES S, DJUKANOVIC V, NICHOLSON M G, WESTA, BIDNEY D, FALCO S C, JANTZ D et al., (2010). Heritable targeted mutagenesis in maize using a designed endonuclease. Plant $J$ 61: 176-187.

HANIN M, VOLRATH S, BOGUCKI A, BRIKER M, WARD E PASZKOWSKI J (2001). Gene targeting in Arabidopsis. Plant J 28: 671-677.

HARTUNG F, SUER S, PUCHTAH (2007). Two closely related RecQ helicases have antagonistic roles in homologous recombination and DNA repair in Arabidopsis Thaliana. Proc Natl Acad Sci USA 104: 18836-18841.

JACQUIERA, DUJON B (1985). An intron-encoded protein is active in a gene conversion process that spreads an intron into a mitochondrial gene. Cell 41: 383-394.

KIRIK A, PECINKA A, WENDELER E, REISS B (2006). The chromatin assembly factor subunit FASCIATA1 is involved in homologous recombination in plants. Plant Cell 18: 2431-2442.

KIRIK A, SALOMON S, PUCHTA H (2000). Species-specific double-strand break repair and genome evolution in plants. EMBO J 19: 5562-5566.

KNOLL A, HIGGINS J D, SEELIGER K, REHA SJ, DANGEL N J, BAUKNECHT M, SCHROPFER S, FRANKLIN F C, PUCHTA H (2012). The Fanconi anemia ortholog FANCM ensures ordered homologous recombination in both somatic and meiotic cells in Arabidopsis. Plant Cell 24: 1448-1464.

LI T, LIU B, SPALDING M H, WEEKS D P, YANG B (2012). High-efficiency TALENbased gene editing produces disease-resistant rice. Nat Biotechno/ 30: 390-392.

LI, J.F., NORVILLE, J.E., AACH, J., MCCORMACK, M., ZHANG, D., BUSH, J., $\mathrm{CHURCH}$, G.M. and SHEEN, J. (2013). Multiplex and homologous recombinationmediated genome editing in Arabidopsis and Nicotiana benthamiana using guide RNA and Cas9. Nat Biotechnol 31: 688-691.

LIEBERMAN-LAZAROVICH M, LEVY A A (2011). Homologous recombination in plants: an antireview. Methods Mol Biol 701: 51-65.

LLOYD A, PLAISIER C L, CARROLL D, DREWS G N (2005). Targeted mutagenesis using zinc-finger nucleases in Arabidopsis. Proc NatlAcad Sci USA 102:2232-2237.

MANNUSSA, DUKOWIC-SCHULZE S, SUER S, HARTUNG F, PACHER M, PUCHTA $H$ (2010). RAD5A, RECQ4A, and MUS81 have specific functions in homologous recombination and define different pathways of DNA repair in Arabidopsis Thaliana. Plant Cell 22: 3318-3330.

MARTON, I., HONIG, A., OMID, A., DE COSTA, N., MARHEVKA, E., COHEN, B., ZUKER, A. AND VAINSTEIN, A. (2013). From Agrobacterium to viral vectors: 
genome modification of plant cells by rare cutting restriction enzymes. Int. J. Dev. Biol. 57: 639-650 [DOI: 10.1387/ijdb.130205av].

MUSSOLINO C, CATHOMEN T (2013). RNA guides genome engineering. Nat Biotechnol 31: 208-209.

NEKRASOV, V., STASKAWICZ, B., WEIGEL, D., JONES, J.D. and KAMOUN, S. (2013). Targeted mutagenesis in the model plant Nicotiana benthamiana using Cas9 RNA-guided endonuclease. Nat Biotechnol 31: 691-693.

NISHIZAWA-YOKOI A, NONAKA S, SAIKA H, KWON Y I, OSAKABE K, TOKI S (2012). Suppression of Ku70/80 or Lig4 leads to decreased stable transformation and enhanced homologous recombination in rice. New Phytol 196: 1048-1059.

OFFRINGA R, DE GROOT M J, HAAGSMAN H J, DOES M P, VAN DEN ELZEN P J, HOOYKAAS P J (1990). Extrachromosomal homologous recombination and gene targeting in plant cells after Agrobacterium mediated transformation. EMBO J 9: 3077-3084.

ONO A, YAMAGUCHI K, FUKADA-TANAKA S, TERADA R, MITSUI T, IIDA S (2012). A null mutation of ROS1a for DNA demethylation in rice is not transmittable to progeny. Plant J. 71: 564-574.

OREL N, KYRYK A, PUCHTA H (2003). Different pathways of homologous recombination are used for the repair of double-strand breaks within tandemly arranged sequences in the plant genome. Plant J 35: 604-612.

OSAKABE K, OSAKABE Y, TOKI S (2010). Site-directed mutagenesis in Arabidopsis using custom-designed zinc finger nucleases. Proc Natl Acad Sci USA 107: 12034-12039.

PAQUESF, HABER JE (1999). Multiple pathways of recombination induced by doublestrand breaks in Saccharomyces cerevisiae. Microbiol Mol Biol Rev 63: 349-404.

PASZKOWSKI J, BAUR M, BOGUCKI A, POTRYKUS I (1988). Gene targeting in plants. EMBO J 7: 4021-4026.

PUCHTA H (1998). Repair of genomic double-strand breaks in somatic plant cells by one-sided invasion of homologous sequences. Plant J. 13: 331-339.

PUCHTA H (2002). Gene replacement by homologous recombination in plants. Plant Mol Biol 48: 173-182.

PUCHTA H (2005). The repair of double-strand breaks in plants: mechanisms and consequences for genome evolution. J Exp Bot 56: 1-14.

PUCHTA H, DUJON B, HOHN B (1993). Homologous recombination in plant cells is enhanced by in vivo induction of double strand breaks into DNA by a site-specific endonuclease. Nucleic Acids Res 21: 5034-5040.

PUCHTA H, DUJON B, HOHN B (1996). Two different but related mechanisms are used in plants for the repair of genomic double-strand breaks by homologous recombination. Proc Natl Acad Sci USA 93: 5055-5060.

QI Y, ZHANG Y, ZHANG F, BALLER J A, CLELAND S C, RYU Y, STARKER C G, VOYTAS D F (2013). Increasing frequencies of site-specific mutagenesis and gene targeting in Arabidopsis by manipulating DNA repair pathways. Genome Res 23: 547-554.

REISSB, SCHUBERTI, KOPCHENK, WENDELERE, SCHELLJ, PUCHTAH (2000). RecA stimulates sister chromatid exchange and the fidelity of double-strand break repair, but not gene targeting, in plants transformed by Agrobacterium. Proc Natl Acad Sci USA 97: 3358-3363.

RENSING S A, LANG D, ZIMMER A D, TERRY A, SALAMOV A, SHAPIRO H, NISHIYAMA T, PERROUD P F, LINDQUIST E A, KAMISUGI Y et al., (2008). The Physcomitrella genome reveals evolutionary insights into the conquest of land by plants. Science 319: 64-69.

RONG Y S, GOLIC K G (2000). Gene targeting by homologous recombination in Drosophila. Science 288: 2013-2018.

ROTH N, KLIMESCH J, DUKOWIC-SCHULZE S, PACHER M, MANNUSS A, PU$\mathrm{CHTA} \mathrm{H}$ (2012). The requirement for recombination factors differs considerably between different pathways of homologous double-strand break repair in somatic plant cells. Plant J 72: 781-790.

SALOMON S, PUCHTA H (1998). Capture of genomic and T-DNA sequences during double-strand break repair in somatic plant cells. EMBO J 17: 6086-6095.

SCHAEFERD G, ZRYD JP (1997). Efficient gene targeting in the moss Physcomitrella patens. Plant J 11: 1195-1206.

SHAKED H, MELAMED-BESSUDO C, LEVY A A (2005). High-frequency gene targeting in Arabidopsis plants expressing the yeast RAD54 gene. Proc Natl Acad Sci USA 102: 12265-12269

SHAN, Q., WANG, Y., LI, J., ZHANG, Y., CHEN, K., LIANG, Z., ZHANG, K., LIU, J., XI, J.J., QIU, J.L. et al. (2013). Targeted genome modification of crop plants using a CRISPR-Cas system. Nat Biotechnol 31: 686-688.

SHUKLA V K, DOYON Y, MILLER J C, DEKELVER R C, MOEHLE E A, WORDEN S E, MITCHELL J C, ARNOLD N L, GOPALAN S, MENG X et al., (2009). Precise genome modification in the crop species Zea mays using zinc-finger nucleases. Nature 459: 437-441.

SIEBERT R, PUCHTA H (2002). Efficient repair of genomic double-strand breaks by homologous recombination between directly repeated sequences in the plant genome. Plant Cell 14: 1121-1131.

SMITHJ, BIBIKOVAM, WHITBYFG, REDDYAR, CHANDRASEGARANS, CARROLL $D$ (2000). Requirements for double-strand cleavage by chimeric restriction enzymes with zinc finger DNA-recognition domains. Nucleic Acids Res 28: 3361-3369.

STREPP R, SCHOLZ S, KRUSE S, SPETH V, RESKI R (1998). Plant nuclear gene knockout reveals a role in plastid division for the homolog of the bacterial cell division protein FtsZ, an ancestral tubulin. Proc NatlAcad Sci USA 95: 4368-4373.

STROTBEK, C., KRINNINGER, S. and FRANK, W. (2013). The moss Physcomitrella patens: methods and tools from cultivation to targeted analysis of gene function. Int. J. Dev. Biol. 57: 553-564.

TERADAR, URAWAH, INAGAKIY, TSUGANE K, IIDAS (2002). Efficient gene targeting by homologous recombination in rice. Nature biotechnology 20: 1030-1034.

THOMAS K R, CAPECCHI M R (1987). Site-directed mutagenesis by gene targeting in mouse embryo-derived stem cells. Cell 51: 503-512.

TOWNSEND J A, WRIGHT D A, WINFREY R J, FU F, MAEDER M L, JOUNG J K, VOYTASDF (2009). High-frequency modification of plant genes using engineered zinc-finger nucleases. Nature 459: 442-445.

TZFIRAT, FRANKMAN LR, VAIDYAM, CITOVSKY V (2003). Site-specific integration of Agrobacterium tumefaciens T-DNA via double-stranded intermediates. Plant Physiol 133: 1011-1023.

VOYTAS D F (2013). Plant Genome Engineering with Sequence-Specific Nucleases Annu Rev Plant Biol. 64: 327-350.

WATERWORTH W M, DRURY G E, BRAY C M, WEST CE (2011). Repairing breaks in the plant genome: the importance of keeping it together. New Phytol 192: 805-822.

WRIGHTDA, TOWNSEND JA, WINFREYRJ, IRWINPA, RAJAGOPALJ, LONOSKY P M, HALL B D, JONDLE M D, VOYTAS D F (2005). High-frequency homologous recombination in plants mediated by zinc-finger nucleases. Plant J. 44:693-705.

XIE, K. and YANG, Y. (in press). RNA-guided Genome Editing in Plants Using A CRISPR-Cas System. Mol Plant doi: 10.1093/mp/sst119

ZHANG F, MAEDERML, UNGER-WALLACE E, HOSHAWJP, REYOND, CHRISTIAN M, LI X, PIERICK C J, DOBBS D, PETERSON T et al., (2010). High frequency targeted mutagenesis in Arabidopsis Thaliana using zinc finger nucleases. Proc Natl Acad Sci USA 107: 12028-12033.

ZHANG Y, ZHANG F, LI X, BALLER J A, QI Y, STARKER C G, BOGDANOVE A J, VOYTAS D F (2013). Transcription activator-like effector nucleases enable efficien plant genome engineering. Plant Physiol 161: 20-27. 


\section{Further Related Reading, published previously in the Int. J. Dev. Biol.}

Female gametophytic mutants of Arabidopsis thaliana identified in a gene trap insertional mutagenesis screen

Vladimir B. Brukhin, Miloslawa Jaciubek, Arturo Bolaños Carpio, Vera Kuzmina and Ueli Grossniklaus

Int. J. Dev. Biol. (2011) 55: 73-84

Genetic, molecular and cellular approaches to the analysis of maize embryo development

Matilde José-Estanyol, Ignacio López-Ribera, Miriam Bastida, Torben Jarhmann, Nuria Sánchez-Pons, Cristian Becerra, Carlos M. Vicient and Pere Puigdomènech Int. J. Dev. Biol. (2009) 53: 1649-1654

Analysis of a homologue of the adducin head gene which is a potential target for the Dictyostelium STAT protein Dd-STATa

Ryota Aoshima, Rieko Hiraoka, Nao Shimada and Takefumi Kawata

Int. J. Dev. Biol. (2006) 50: 523-532

Gametophyte interaction and sexual reproduction: how plants make a zygote Leonor C. Boavida, Ana Maria Vieira, Jörg D. Becker and José A. Feijó Int. J. Dev. Biol. (2005) 49: 615-632

Plant microRNAs and development

Sara Jover-Gil, Héctor Candela and María-Rosa Ponce Int. J. Dev. Biol. (2005) 49: 733-744

5 yr ISI Impact Factor $(2011)=2.959$
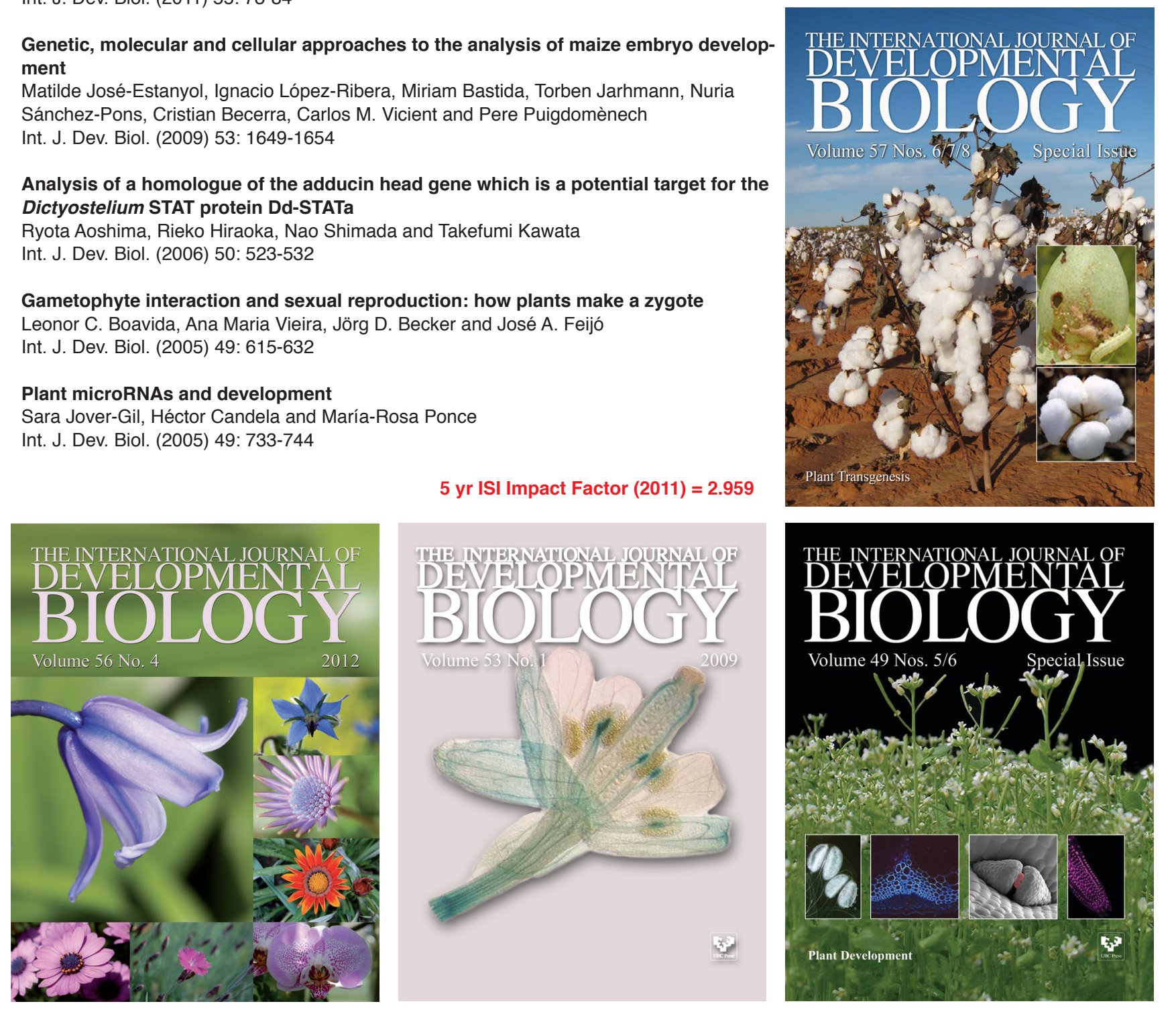